\title{
Research Article \\ COMPARISON OF 24-HOUR I-131 THYROID UPTAKE BY GAMMA CAMERA USING HIGH ENERGY COLLIMATOR WITH STANDARD THYROID UPTAKE PROBE BASED METHOD
}

\author{
DESWAL S., SINGH D.K.* AND BHATT D.C. \\ Dr Ram Manohar Lohia Institute of Medical Sciences, Lucknow, Uttar Pradesh 226010, India \\ *Corresponding Author: Email - dkpalam@gmail.com
}

Received: June 20, 2018; Revised: June 26, 2018; Accepted: June 27, 2018; Published: June 30, 2018

\begin{abstract}
The aim and objective of this study were to the made comparison of 24-hour I-131 thyroid uptake calculated by Gamma camera-based method and thyroid uptake probe based method after administration of a diagnostic l-131 solution in patients with thyroid disease. Methods: This study group was comprised of 25 patients, out of which 10 patients were male, and 15 patients were female (Age range 20-62 yrs). In these patients had benign thyroid disorders and most of who had been referred for Thyroid evaluation before radioiodine-131 treatment. A liquid form of 131 ( $(50 \mu \mathrm{Ci}$ [1.85MBq]) was administered to the patient with adequate flushing of the vial to ensure maximum oral administration of the dose. The dose was prepared with the help of pipetting technique. Radioiodine-131 uptake was usually measured at $25 \mathrm{~cm}$ distance from the face of the crystal to the anterior neck or phantom and also 24 hours thyroid uptake was calculated by using a probe-based method and a camera-based method with a high-energy parallel-hole (HEGP) collimator. Gamma camera uptake calculated by two method syringe method and decay correction method for later method first correction factor derived and then applies it. Result and Discussion: Radioiodine uptake was measured by gamma camera method and thyroid uptake value was not equal of that measured by thyroid uptake probe when we use syringe method. Therefore, by applying a study specific correction factor in gamma camera uptake value and then we can equate the uptake value of probe method and gamma camera method. But when we apply correction factor in gamma camera method then the uptake values with the help of gamma camera are very close to the uptake value obtain by thyroid probe system. Conclusion: On the basis of precision in counting by thyroid uptake probe it takes the edge over gamma camera method but with the help of study specific correction factor the Gamma camera based method is a good substitute for the thyroid probe based method and the camera-based thyroid uptake method can be substituted well for the probe based method. Nuclear Medicine Centre that do not have a dedicated thyroid probe can use this method to obtain quantitative information in a single scan and thyroid uptake probe method can be replaced by gamma camera method
\end{abstract}

Keywords- I-131, High Energy Collimator, Thyroid Uptake Probe, Gamma Camera, pertechnetate

Citation: Deswal S., et al., (2018) Comparison of 24-Hour l-131 Thyroid Uptake by Gamma Camera Using High Energy Collimator with Standard Thyroid Uptake Probe Based Method. International Journal of Microbiology Research, ISSN: 0975-5276 \& E-ISSN: 0975-9174, Volume 10, Issue 6, pp.-1274-1278.

Copyright: Copyright@2018 Deswal S., et al., This is an open-access article distributed under the terms of the Creative Commons Attribution License, which permits unrestricted use, distribution and reproduction in any medium, provided the original author and source are credited.

\section{Introduction}

The era of clinical nuclear medicine began with the radioiodine uptake test. In1940, Hamilton reported the first external measurements of radioiodine uptake in the thyroid by means of Geiger counter [1]. The 24-hour RAIU test was introduced in 1950 by Werner, et al. as a method to appraise thyroid function [2]. Radioiodine (I-131) therapy is an effective and well-tolerated option in patients with hyperthyroidism (thyrotoxicosis) due to Graves' disease and thyrotoxic multinodular goiter and in certain cases of non-thyrotoxic multinodular goiter [3-6]. Radiotherapy with radioactive iodine-131has been used to treat benign thyroid diseases for over 50years [7]. Conditions associated with elevated or normal uptake can be distinguished from those associated with near-absent uptake (e.g. painless postpartum thyroiditis, subacute thyroiditis ,or ingestion of thyroid hormone) [3-6].The thyroid scan in this clinical context helps especially in determining the presence of nodularity: the uptake pattern in solitary thyrotoxic adenoma generally is focal in the adenoma and suppressed in the surrounding and contralateral thyroid tissue, whereas in thyrotoxic multinodular goiter there are multiple areas of focally enhanced and suppressed uptake.Althoughl-123 is preferred for diagnostic scanning, -131 continues to be used in several developing nations where iodine- 123 is currently not available [8]. Radioiodine thyroid uptake measurement test is a commonly performed in daily nuclear medicine practice [9]. Although most academic medical centers estimate thyroid uptake by using a thyroid uptake probe (which is ideal), some do not have this instrument and hence use pertechnetate( $99 \mathrm{mTcO} 4)$ scanning with a gamma camera-based method.99mTcO4 is not organified but trapped by the thyroid gland. This can result in a small range of normal uptake and high background activity, but totalbody radiation exposure is less because $99 \mathrm{mTc}$ does not have a beta component. In addition, in nodular disease there may be discordance between radioiodine and $99 \mathrm{mTc}$ images both have different physiologic uptake mechanism. For patients with differentiated thyroid cancer, the usually administered activity is $37-185 \mathrm{MBq}$ $(1-5 \mathrm{mCi})$ of I-131orally, and the neck and entire body are imaged at 48-96 h [10]. The aim of this study was made a direct comparison of 24 hour I-131 uptake in the same patient using a camera-based method using high energy collimator and a thyroid uptake probe based method in thyroid diseases after administration of a $1.85 \mathrm{MBq}$ l-131 Sodium lodide Solution.

\section{Material and Method \\ Design Overview}

This prospective study was performed to compare the gamma camera method and standard probe-based method for radioiodine thyroid uptake. The study was carried out in the department of nuclear medicine at DRRMLIMS, Lucknow, from December 2015 to June 2017 after written informed consent was taken from the participants. Maximum patients coming into department were for thyroid gland evaluation. 
The consent forms and study procedure were reviewed and approved by the institutional ethics committee.

Inclusion Criteria: Adult patients with a clinical diagnosis of benign thyroid disorder.

Exclusion criteria: Patients who were having younger than 18years, taking Antithyroid drug within seven days and in case of female patients, if she was pregnant and or lactating and others patients who did not give written consent for the study.

If any one of the patients was suffering from deranged TSH level, a preliminary clinical workup was performed, the previous history of the thyroid was recorded history of hypertension, history of LMP, and current blood report of TSH and detail of previous treatment was received.

\section{Method for the preparation of $1.85 \mathrm{MBq}$ ( 50 microcurie) dose ofiodine-131:}

Dose preparation was done with the help of pipetting technique. It is based on the principle of dilution.

Patient Information: Thyroid studies must be interpreted in light of the patient's clinical history, serum thyroid function studies, and findings at thyroid palpation. Patient preparation: The standard precaution for Radioiodine uptake and scanning were taken with regards to food and medications that interfere with radionuclide thyroid studies and pregnant and lactating mothers were excluded from this study. All the Patients should have nothing by mouth for approximately 4 hours before radioiodine ingestion to ensure good absorption with no restriction on the normal intake of water. $1.85 \mathrm{MBq}$ (50 microcurie) of iodine-131 prepared with the help of pipetting technique.

\section{Thyroid Uptake:}

\section{Radioactive lodine Percent Uptake}

I-131Sodium lodide solution was used for calculation of the \% RAIU. Room back ground activity is first determined. The radioiodine in liquid form inside the vial with known calibrated activity is placed in a Lucite neck phantom and counts are obtained with the detector placed at a standardized distance of $25 \mathrm{~cm}$. After that pre administered syringe count take under gamma camera with the matrix of $128 \times 128$, zoom of 2 for 60 seconds and then radioiodine dose was administered to the patient. After that post administered syringe count take under the gamma camera. Both counts taken at $25 \mathrm{~cm}$ distance from detector and acquisition time for each is 60 seconds. The patient's thigh (background) was also counted for the background correction. To calculate the \% RAIU, counts are obtained for the patient's neck and thigh (for background). The percent radioiodine uptake is calculated according to the formula:

$R A I U=\frac{\text { Neck Count }(\mathrm{cpm})-\text { Thigh Count }(\mathrm{cpm})[\text { background corrected }]}{\text { Administered Count }(\mathrm{cpm})-\text { BackgroundCount }(\mathrm{cpm})[\text { decayandbackground corrected }}$

After 24 hours patients were positioned for thyroid scan under gamma camera at distance $25 \mathrm{~cm}$ away from the detector. The matrix of $128 \times 128$ for 5 minute or 300 $\mathrm{k}$ count is fixed for thyroid scan. Patient neck extended and pillow below the shoulder for support is placed. Gamma Camera uptake calculated with the help of software thyroid index available with GE Manufactured dual head Gamma Camera (Discovery NM/CT 670) in our department. RAIU calculation with camera based method done by two methods one with decay correction method and another without decay corrected method.

\section{Without decay correction method}

First, drawn ROI on thyroid scan image and determine background near the thyroid gland after that with the help of thyroid Index software we calculated the radioiodine uptake.

\section{Decay correction method}

In this method first, we established a decay correction factor for24-hour activity, then entered the values in the thyroid Index software and calculated the radioiodine uptake.

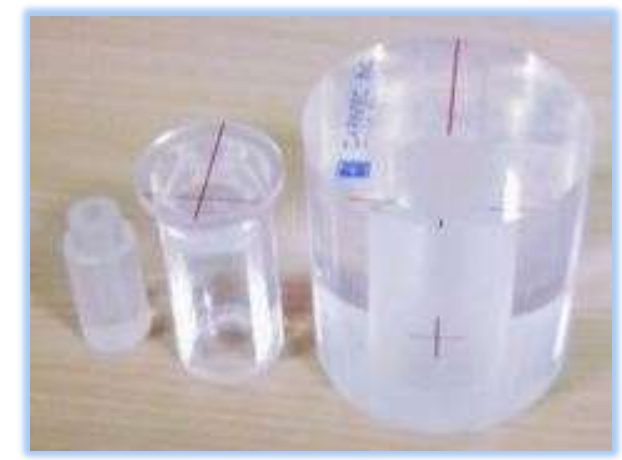

Fig-1 Lucite Neck Phantom

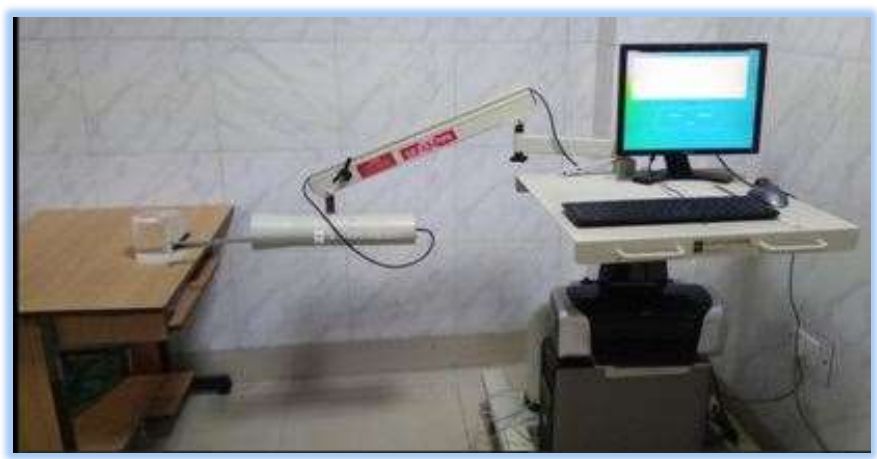

Fig-2 Thyroid Uptake Probe

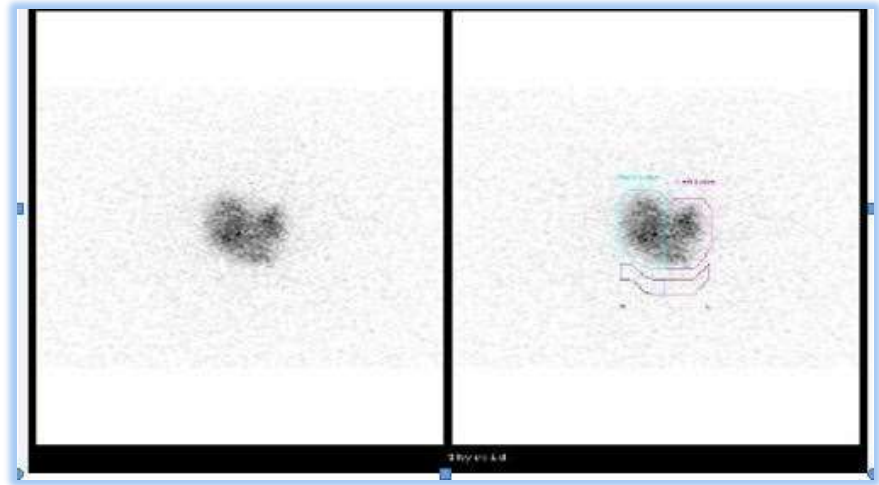

Fig-3 Thyroid Scan using Gamma Camera. This is the Image of Thyroid Scan [After the Scan we drew an ROI on this image and calculated the radioiodine uptake using the software (Xeleris, thyroid uptake index)].

\section{Result}

This study group comprised of 25 patients, out of which 10 were males, and 15 were females. The age range of these patients was from 20 to 62yrs. Most of the patients belonged from the age of 25 to 50 years. The number of female patients to male patient ratio was1.5.The mean age of this group is 39 years. Most of the patients in this group were suffering from the different thyroid disorder. TSH range was 0.005 to $4.40(\mu \mathrm{l} \mathrm{U} / \mathrm{ml})$ and mean of TSH was $0.35(\mu \mathrm{lU} / \mathrm{ml})$. Mean TSH range was meagre which showed most of the patients belonged to Graves' disease. Uptake range in probe-based standard method was $2.3-72.1 \%$ and mean uptake was $41.008 \%$. Uptake range in gamma camera method using syringe method was 0.6-47.8\% and mean uptake was $19.63 \%$. Using decay correction factor 1.0 uptake range in gamma camera method was $1.4-68.4 \%$ and mean uptake was $35.96 \%$.

Using decay correction factor 0.9 Uptake range in gamma camera method was 1.6-75.5 \% and mean uptake was $38.46 \%$. Using decay correction factor 0.87 uptake range in gamma camera method was1.7-78.5 \% and mean uptake was $40.17 \%$. After comparing these all methods with each other, we found that the about fifty percent of mean uptake value of standard probe based method and by applying statistical analysis, we derived a relation, i.e., correction factor between probe uptake, and gamma camera uptake and equate the uptake values of both, i.e. the gamma camera syringe method and probe based uptake value.

$$
P=(A+0.379) / 0.478
$$




\section{$P=$ probe uptake percent}

$A=$ gamma camera uptake percent (by syringe method)

Mean uptake of gamma camera decay correction method using decay correction factor of 1and 0.9 was near about to the mean uptake value of the standard probe-based method. However, mean uptake of gamma camera-based decay correction method using decay correction factor of 0.87 was very close to mean uptake value of standard probe-based method.

Table -1 Age wise distribution of patients

\begin{tabular}{|c|c|c|}
\hline Age in year & Number of patient & Percentage (\%) \\
\hline $21-30$ & 7 & 28 \\
\hline $31-40$ & 6 & 24 \\
\hline $41-50$ & 9 & 36 \\
\hline$>50$ & 3 & 12 \\
\hline Total & 25 & 100 \\
\hline
\end{tabular}

Patient's most common Age range was from 41 to 50 years, and about $36 \%$ belong to this range, and minimum belong to the age of $>50 y r s$.

Standard deviation between probe uptake method and gamma camera decay method was very less. Therefore, gamma camera method with Correction Factor (C.F) of 0.87 can substitute for the probe based method. However, there was the significant difference in different uptake methods. Repeated measure, Statistical analysis was done by using Analysis of variance (ANOVA)and pair t-test of different uptake method. There is significant correlation between probe-based method) and gamma camera method with C.F of 0.87 ).

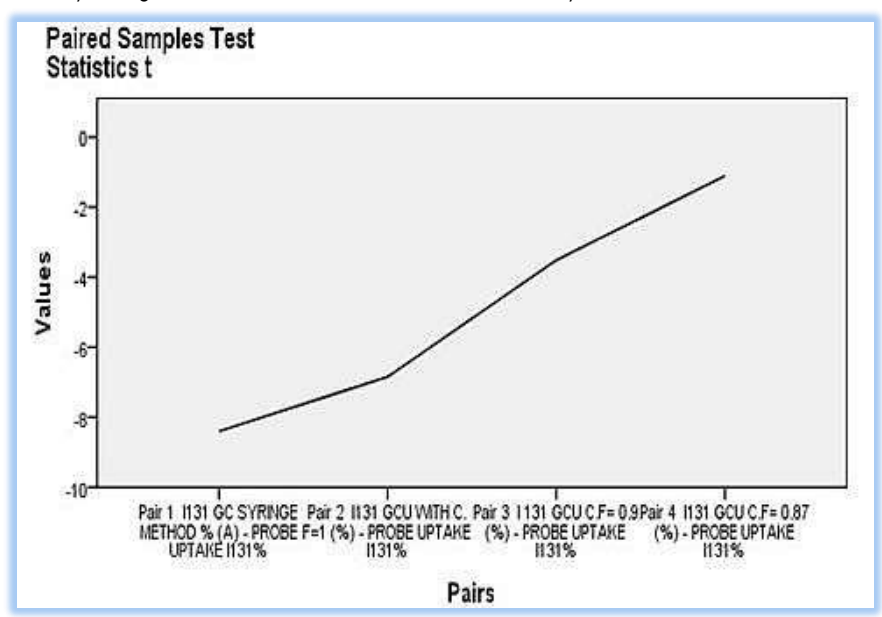

In this graphical representation each test comparing with the gold standard probebased method and pair 4 (probe-based method and gamma camera based method withC.Fof0.87) showed linear relations.

\section{Discussion}

Developments of the new radiopharmaceutical for thyroid scintigraphy, such as I$123,99 \mathrm{mTc} 04$, and I-131 leads to evaluate thyroid function before radioiodine therapy. For thyroid uptake and scintigraphic study most preferred radiopharmaceutical is lodine-123 because it has low gamma energy and less half-life, hence the patient's dose is low compared to others, but because of its limited availability and higher cost, it is hardly used in nuclear medicine centers. lodine-131 is the best substitute for lodine-123 because of its low cost and easy availability, but lodine-131 comprises high dosimetry and gives the poor quality image. After administration of lodine-131 recommended time for uptake measurement is approximately 24 hours and the standard instrument is thyroid uptake probe in the case when thyroid uptake probe is not available then gamma camera with a high energy collimator can be used but the use of standardized neck phantom remains necessary. Those centers which do not have lodine-123 or a thyroid uptake probe, they can use the thyroid scintigraphy for uptake but $99 \mathrm{mTCO}_{4}$ thyroid uptake study is not a substitute for traditional iodine uptake study because 99mTc-pertechnetate doesn't organified in thyroid gland and so it doesn't give the exact functional information as lodine-131 gives $1-123$ is a better substitute for $\mid-131$ because of its favourable physical characteristics [11] for imaging. It gives better imaging and minimum radiation dose to patients, Studies have demonstrated that 4-h 123I uptake can be a substitute for 24-h 131/ uptake $[8,12]$, eliminating the need for patients to revisit the center on the next day. Its availability and cost are the challenges, and this is the reason for its limited use in developing countries. Research studies to this end have been performed by various investigators to validate modifications of the standard recommended procedure. Wasilewski-Radwanska, et al., compared 99mTc04 thyroid uptake measurement measured using a gamma probe and a gamma camera and observed an excellent correlation between them [13]. Calculation of thyroid uptake with the help of thyroid uptake probe is the conventional method. In this study, we have shown that gamma camera based iodine-131 uptake method can be used a substitute for the thyroid uptake probe based method and thyroid uptake values obtained by these two methods correlated well $(P<0.005)$ with each other, for various types of patients. For thyroid uptake test, iodine-131 can be used in two forms, i.e., lodine -131 sodium iodide solution form and iodine-131 capsule form. lodine-131 sodium iodide solution form is widely used instead of capsule because capsules are expensive and don't fulfill the sudden demand of various amount of activity. For capsule, it requires the proper procedure for transport from BRIT. lodine-131 sodium iodide solution is cost-effective and prepared with the help of pipette using dilution technique. It gives the more accurate amount of activity. The reason behind the application of pipette technique is that the dose calibrator cannot give an accurate measurement of activity below $0.5 \mathrm{mci}$ as it becomes difficult to differentiate it from the background. In this study, most of the patients were diagnosed with 'Graves ' disease, some with thyroiditis, nodular goiter, whose TSH had level was from 0.05 to $4.02 \mu \mathrm{lU} / \mathrm{ml}$. The TSH range for Graves' disease was from 0.01 to $4.2 \mu \mathrm{lU} / \mathrm{ml}$. Most of the Graves diseased patients were given low dose iodine therapy. In our study, the thyroid uptake values were calculated mainly by two methods, one with the help of thyroid uptake probe and another with the help of gamma camera method. In the Probe based method, the system itself performs decay correction, so no further decay correction is required, but gamma camera method can be of two categories, one with decay correction method and second without decay correction method or syringe method.

Syringe method: In this method we drew an ROI around the thyroid gland and a semi lunar background, then the calculation of uptake was done automatically with the help of software. By applying statistical analysis, we derived a relationship i.e. correction factor between probe uptake and gamma camera uptake.

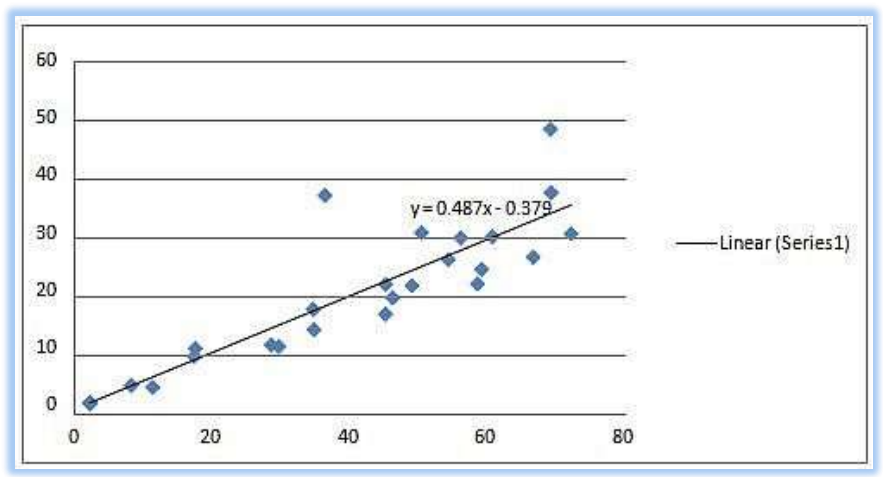

Fig-3 Y-axis shows radioiodine probe based uptake, and $x$-axis shows gamma camera uptake with syringe method

Decay correction factor method: We have software for thyroid uptake calculation. In this software no need of pre and post syringe count. Information needed for this software is done in proper unit, decay correction factor concerning the unit of dose and time of measurement of uptake.

\section{In our study, we applied 3 types of decay correction factors}

In the first type, we assume that the same activity is retained in the patient's body as of previous day although in reality, it is not possible. For this, we apply the decay correction factor of 1 .

The second type, because iodine- 131 decay $10 \%$ per day means $90 \%$ of activity remains after 24 hours and apply a correction factor of 0.9 . 
Table-1 Radioiodine uptake data of patients by using Thyroid probe as well as Gamma Camera based [using different Calibration factor (C.F)]

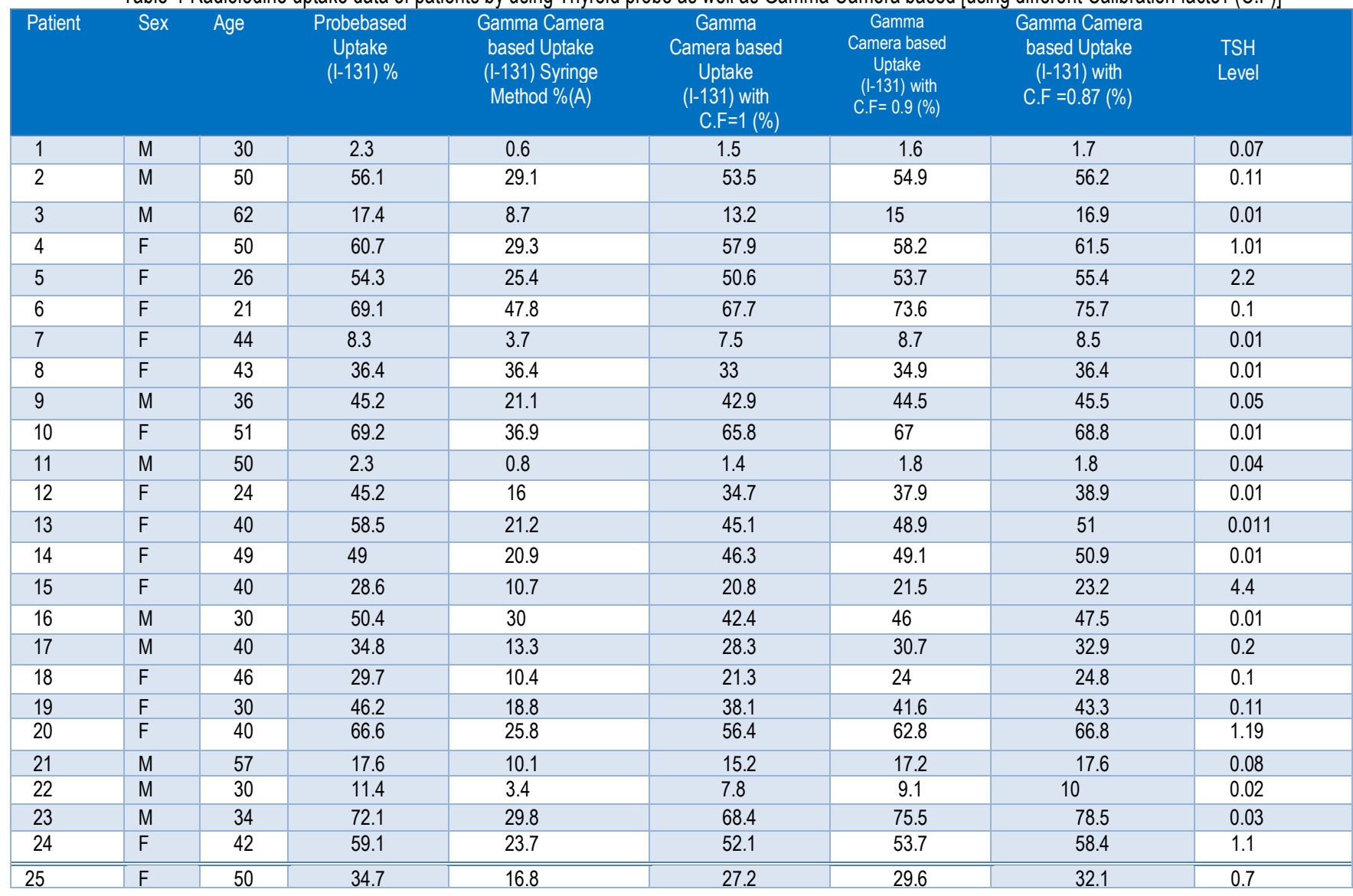

In the third type, we derived a decay correction factor according to the procedure given in the software manual for iodine-123. It requires ROI over thyroid and background and surface area of both and derives a correction factor, which is 0.87 for iodine-131. After applying these all factors for the same patient, according to our results, the gamma camera method using the decay correction factor of 0.87 and 0.9 shows a very strong correlation coefficient of 0.999. Gamma camera method using decay correction factor of 1 and 0.9 also shows a very strong correlation coefficient between them. Gamma camera method using decay correction factor of 1 and 0.87 shows strong correlation but with minor deterioration. Gamma camera method using decay correction factor of 1 and with our reference standard method that is thyroid probe uptake method shows good correlation coefficient with each other of 0.998 . Correlation coefficient between gamma camera method with decay correction factor of 0.87 and thyroid probe uptake is 0.991 , this value is the most reliable correlation value between gamma camera method and thyroid uptake probe method. Hence merely it can be concluded that the gamma camera using decay correction factor of 0.87 shows most robust relation, i.e., highest correlation coefficient with thyroid uptake probe method. Hence, according to our study gamma camera method with decay correction factor of 0.87 can be the best substitute of thyroid uptake probe method, between all of these i.e. gamma camera syringe method (no correction factor) Gamma camera method with decay correction factor of 1 and 0.9 (decay correction). At last, our study strongly recommends that gamma camera method can be a good alternative of thyroid probe system and among the several methods for thyroid uptake gamma camera method with decay correction factor of 0.87 is the best substitute. There are several studies comparing thyroid uptake values by thyroid uptake system at different time intervals but very limited studies have been done comparing thyroid uptake value from thyroid uptake system and gamma camera method. Recently, Biju K. Menon, et al. shows a comparison of I-131 thyroid uptake values between gamma camera method and probe-based method [8]. However, in their study, they did not mention about decay correction factors like we did and also our study well reveals the importance of appropriate decay correction, and this is why we embarked upon this study. We derived a decay correction factor and applied it to the Gamma camera thyroid uptake values and then compared these values with the thyroid uptake values by thyroid uptake system, which shows a very strong correlation coefficient. Wasilewski-Radwanska M Studied the comparison between the data obtain in thyroid $99 \mathrm{mTc}$ uptake measurements with a gamma camera against thyroid probe (scintillation probe) system. They study 30 patients (average age of 52 ) with hyperthyroidism contrary [13]. We studied on 25 patients out of them 20 were hyperthyroidism patient similar to our result they also concluded that gamma probe could be an alternative to the gamma camera as a simple and efficient technique. As discussed above our result dictated that the gamma camera can be a good alternative of scintillation probe so it is very clear from the conclusion of WasilewskaRadwanska $M$ and ours that both of the methods for thyroid uptake i.e. gamma camera method and thyroid uptake probe method can be used alternatively with similar and efficient thyroid uptake value.

\section{Conclusion}

As per our study, camera-based thyroid uptake high energy parallel hole collimator [HEGP] and appropriate decay correction can be substituted well for probe based method. This method can be an alternative of thyroid uptake probe method. Nuclear medicine centers that do not have a dedicated thyroid probe can use the Camera based method to obtain quantitative information in a single scan. However, before the adaptation of camera based method into the clinical routine that individual centers should perform a comparative evaluation and standardization. Also, from our study, it is advisable that the nuclear medicine centers with limited resources need not to buy a dedicated thyroid uptake system, as the results from gamma camera method with appropriate decay correction are also very reliable.

Application of research: nuclear medicine centers with limited resources need not to buy a dedicated thyroid uptake system, as the results from gamma camera method with appropriate decay correction are also very reliable. 
Research Category: Clinical Nuclear Medicine.

\author{
Abbreviations \\ RAIU: Radioiodine Uptake \\ LU: Late Uptake \\ EU: Early Uptake \\ EBF: Early Thyroid Blood Flow \\ ULI : Upper Large Intestine \\ GD : Graves' disease \\ LU: Late Uptake \\ LEAP : Low-Energy All-Purpose \\ PH :Pin Hole Collimator \\ ROI: Region of Interest \\ El: Euthyroid Individuals \\ BKG : Background \\ SD : Standard Deviation \\ C.F : Correction Factor
}

Acknowledgement / Funding: Author thankful to Dr Ram Manohar Lohia Institute of Medical Sciences, Lucknow, Uttar Pradesh 226010, India

*Research Guide or Chairperson of research: Satyawati Deswal

University: Dr Ram Manohar Lohia Institute of Medical Sciences, Lucknow, Uttar Pradesh 226010, India

Research project name or number: MSc Thesis

\title{
Author Contributions: All author equally contributed
}

Author statement: All authors read, reviewed, agree and approved the final manuscript

\section{Conflict of Interest: None declared}

Ethical approval: Approval was taken from Institutional Ethics Committee of $\mathrm{Dr}$ Ram Manohar Lohia Institute of Medical Sciences, Lucknow, Uttar Pradesh 226010, India

Ethical Committee Approval Number: 37/15

\section{References}

[1] Hamilton J.G., solely M.H. (1940) Am J Physiol.,131,135-143, 1940.

[2] Werner S.C., Hamilton H.B., Leifer E., et al., (1950) J. Clin Endocrinol, 10,1054-1076, 1950.

[3] Cooper D.S. (2003) Lancet, 362,459-468.

[4] Pearce E.N. (2006) BMJ, 332,1369-1373.

[5] Biondi B., Cooper D.S. (2008) Endocr Rev, 29,76-131.

[6] Hallowell J.G., Staehling N.W., Flanders W.D., et al. (2002) J Clin Endocrinol Metab., 87,489-499.

[7] Becker D.V. and Swin C.T. (1996) Sem in Nucl Med., 26(3)155-164.

[8] Biju K. Menon, Ramesh D. Rao, Amit Abhyankar, M.G. Ramakrishna Rajan, and Sandip Basu (2014) India J Nucl Med Technol, 42,194-197

[9] Kusai M. Al-Mugbel and Reema M. T ashtoush (2010) Jordan J Nucl. Med Technol., 38,32-36.

[10] Balon H.R., Silberstein E.B., Meier D.A., et al., (2006) Society of Nuclear Medicine Procedure guideline for thyroid uptake measurement, version 3.0 .

[11] Harbert J.C. Radioiodine therapy of hyperthyroidism In: Harbert J.C., Eckelman W.C., Neumann R.D., et al. Nuclear medicine: diagnosis and therapy. New York, NY, (1996) Thieme Medical, 959-973.

[12] Society of Nuclear Medicine Procedure Guideline for Thyroid Uptake Measurement (2006).

[13] Wasilewska-Radwanska M. (2006) Polish J. of Environ. Stud., 15, №. $4 \mathrm{~A}, 213-215$. 